\title{
Ray-tracing traveltime tomography versus wave-equation traveltime inversion for near-surface seismic land data
}

\author{
Lei $\mathrm{Fu}^{1}$ and Sherif M. Hanafy ${ }^{2}$
}

\begin{abstract}
Full-waveform inversion of land seismic data tends to get stuck in a local minimum associated with the waveform misfit function. This problem can be partly mitigated by using an initial velocity model that is close to the true velocity model. This initial starting model can be obtained by inverting traveltimes with ray-tracing traveltime tomography (RT) or wave-equation traveltime (WT) inversion. We have found that WT can provide a more accurate tomogram than RT by inverting the first-arrival traveltimes, and empirical tests suggest that RT is more sensitive to the additive noise in the input data than WT. We present two examples of applying WT and RT to land seismic data acquired in western Saudi Arabia. One of the seismic experiments investigated the water-table depth, and the other one attempted to detect the location of a buried fault. The seismic land data were inverted by WT and RT to generate the P-velocity tomograms, from which we can clearly identify the water table depth along the seismic survey line in the first example and the fault location in the second example.
\end{abstract}

\section{Introduction}

For land seismic surveys, the near-surface velocity model is important for computing an accurate migration image (Vermeer, 2002). The near-surface velocity model is obtained by traveltime tomography, which inverts the first-arrival traveltimes for the P-velocity model. These arrivals are either diving waves or refractions. Ray-tracing traveltime tomography (RT) has been used for earthquake imaging of the earth's interior from the early 1970s (Aki et al., 1977; Humphreys et al., 1984), which was mostly implemented using the high-frequency approximation of ray tracing to calculate the traveltimes. To avoid the high-frequency assumption of RT, Luo and Schuster (1991a, 1991b) propose a wave-equation traveltime (WT) inversion method to obtain the near-surface Pwave velocity model. The WT method can be more accurate than RT, but it is at least an order of magnitude more costly because the wave equation must be numerically solved for each source.

Full-waveform inversion (FWI) attempts to solve an underdetermined system of equations, in which many different models yield synthetic data that match the observed data within a reasonable tolerance. The local minima and cycle-skipping problems in FWI are partly caused by an inaccurate estimate of the source wavelet, an inaccurate amplitude modeling, elastic effects, random noise, and an inaccurate initial velocity model.
To suppress the source-wavelet effect, Frazer and Sun (1998) develop a source-independent algorithm using convolved wavefields. Bai et al. (2014) present viscoacoustic waveform inversion in the time domain for velocity estimation, in which viscoacoustic-wave equations are used to generate accurate amplitudes. To reduce the harmful effects of noise in the data, Neelamani et al. (2008) apply a curvelet-based algorithm to attenuate random and coherent linear noise in a 3D data set from a carbonate environment. To partly migrate the local minima problem of FWI, Yu and Hanafy (2014) apply a multiscale early arrival waveform inversion (MEWI, which gradually lengthens the time window for muting early arrivals) to shallow seismic land data. However, an accurate initial velocity model is very important for obtaining an accurate tomogram in MEWI and FWI. The WT and RT inversion methods can provide a reasonable initial velocity model. However, WT (Luo and Schuster, 1991b) does not require a high-frequency assumption and can account for body-wave, diffraction, reflection, and head-wave traveltimes. Hence, the WT (Luo and Schuster, 1991a, 1991b) method should provide a more accurate initial velocity model than the RT method for MEWI or FWI.

In this paper, one synthetic and two field-data examples are used to demonstrate the advantages of WT over the RT method. We first describe the theory of the WT

\footnotetext{
${ }^{1}$ King Abdullah University of Science and Technology (KAUST), Division of Physical Science and Engineering, Thuwal, Saudi Arabia. E-mail: lei .fu@kaust.edu.sa.

${ }^{2}$ King Abdullah University of Science and Technology (KAUST), Division of Physical Science and Engineering, Thuwal, Saudi Arabia and Cairo University, Geophysics Department, Thuwal, Saudi Arabia. E-mail: sherif.mahmoud@kaust.edu.sa.

Manuscript received by the Editor 18 November 2016; revised manuscript received 13 April 2017; published ahead of production 10 May 2017 ; published online 06 July 2017. This paper appears in Interpretation, Vol. 5, No. 3 (August 2017); p. SO11-SO19, 12 FIGS., 1 TABLE.

http://dx.doi.org/10.1190/INT-2016-0210.1. @ 2017 Society of Exploration Geophysicists and American Association of Petroleum Geologists. All rights reserved.
} 
and RT methods, and then we describe how WT and RT are implemented using a workflow. We then present the numerical results for applying WT and RT to one synthetic and two field-data examples. The final sections present the discussion and conclusion.

\section{Wave-equation traveltime inversion}

For seismic data, refraction waves can be modeled by solving the $2 \mathrm{D}$ constant-density acoustic-wave equation (Yilmaz, 2001):

$$
\nabla^{2} p(\mathbf{x}, t)-\frac{1}{\mathbf{v}(\mathbf{x})^{2}} \frac{\partial^{2} p(\mathbf{x}, t)}{\partial t^{2}}=f(\mathbf{x}, t),
$$

where $\mathbf{x}$ is the model space, $\nabla^{2}=\left(\left[\partial^{2} / \partial x^{2}\right],\left[\partial^{2} / \partial y^{2}\right]\right)$ is the 2D Laplacian, $\mathbf{v}(\mathbf{x})$ is the P-wave velocity model, $p(\mathbf{x}, t)$ is the pressure field, and $f(\mathbf{x}, t)$ is the source term. The WT method is designed to minimize the following objective function:

$$
\Phi(\mathbf{v})=\frac{1}{2}\left\|\Delta \tau_{r s}\right\|^{2},
$$

where $\Delta \tau_{r s}=\tau_{\text {cal }}\left(\mathbf{x}_{r}, \mathbf{x}_{s}\right)-\tau_{\text {obs }}\left(\mathbf{x}_{r}, \mathbf{x}_{s}\right)$ is the refraction traveltime residual for a source at $\mathbf{x}_{s}$ and a receiver at $\mathbf{x}_{r}$ (Luo and Schuster, 1991a, 1991b). In practice, the traveltime difference can be found by crosscorrelating the predicted and the observed seismograms (Luo and Schuster, 1991b). The P-velocity distribution can be iteratively updated using any gradient-based method (Scales, 1985), such as the conjugate-gradient method:

$$
\mathbf{v}_{k+1}=\mathbf{v}_{k}+\alpha_{k} \mathbf{d}_{k},
$$

where $\mathbf{v}_{k+1}$ is the P-velocity model at the $(k+1)$ th iteration; $\alpha_{k}$ is the scale step length, which can be found using a line-search algorithm; and $\mathbf{d}_{k}$ is the updated direction:

$$
\begin{gathered}
\mathbf{d}_{k}=-\mathbf{g}_{k}+\beta_{k} \mathbf{d}_{k-1}, \\
\beta_{k}=\frac{\left(\mathbf{g}_{k}-\mathbf{g}_{k-1}, \mathbf{g}_{k}\right)}{\left(\mathbf{g}_{k-1}, \mathbf{g}_{k-1}\right)},
\end{gathered}
$$

Here, $\beta_{k}$ is a scalar chosen to insure that $\mathbf{d}_{k}$ is conjugate to $\mathbf{d}_{k-1}$, the current search direction (Wright and Nocedal, 1999) $\mathbf{d}_{k}$ is given by the weighted summation of the current $k$ th gradient $\mathbf{g}_{k}=\left(\partial \Phi / \partial \mathbf{v}_{k}\right)$ and the former search direction $\mathbf{d}_{k-1}$. Because WT is a nonlinear inversion method, we use a quadratic line-search method to compute the step length (Wright and Nocedal, 1999). Several reasonable values of the step length are selected, e.g., $\alpha_{1}=$ 0.5 and $\alpha_{2}=1$, and the objective functions $\Phi\left(\mathbf{v}_{k}+\alpha_{1} \mathbf{d}_{k}\right)$ and $\Phi\left(\mathbf{v}_{k}+\alpha_{2} \mathbf{d}_{k}\right)$ are computed to determine if they are less than $\Phi\left(\mathbf{v}_{k}\right)$, and if $\Phi\left(\mathbf{v}_{k}+\alpha_{1} \mathbf{d}_{k}\right)<\Phi\left(\mathbf{v}_{k}+\alpha_{2} \mathbf{d}_{k}\right)$. Then, the following quadratic formula is used to determine the interpolated value of the objective function:

$$
\begin{aligned}
\Phi\left(\mathbf{v}_{k}+\alpha \mathbf{d}_{k}\right)= & \frac{\left(\alpha-\alpha_{1}\right)\left(\alpha-\alpha_{2}\right)}{\alpha_{1} \alpha_{2}} \Phi\left(\mathbf{v}_{k}\right) \\
& +\frac{\alpha\left(\alpha-\alpha_{2}\right)}{\alpha_{1}\left(\alpha_{1}-\alpha_{2}\right)} \Phi\left(\mathbf{v}_{k}+\alpha_{1} \mathbf{d}_{k}\right) \\
& +\frac{\alpha\left(\alpha-\alpha_{1}\right)}{\alpha_{2}\left(\alpha_{2}-\alpha_{1}\right)} \Phi\left(\mathbf{v}_{k}+\alpha_{2} \mathbf{d}_{k}\right) 0 \leq \alpha \leq \alpha_{2} .
\end{aligned}
$$

The scalar value $\alpha$ is found by differentiating $\Phi(\alpha)$ equation 6 with respect to $\alpha$, setting the result to zero, and solving for $\alpha$ (for further details, see Schuster, 2017).

In WT inversion, the gradient of the misfit function with respect to the P-wave velocity is given by (Luo and Schuster, 1991b)

$$
\mathbf{g}=\frac{1}{(\mathbf{v})^{3}} \sum_{s} \int p\left(\mathbf{x}, t \mid \mathbf{x}_{s}\right)_{\text {cal }} p\left(\mathbf{x}, t \mid \mathbf{x}_{r}\right) d t,
$$

where

$$
\dot{p}\left(\mathbf{x}, t \mid \mathbf{x}_{s}\right)=\sum_{r} G\left(\mathbf{x},-t \mid \mathbf{x}_{r}, 0\right) * \delta \tau\left(\mathbf{x}_{r}, t \mid \mathbf{x}_{s}\right),
$$

and the symbol $*$ represents temporal convolution. The function $p\left(\mathbf{x}, t \mid \mathbf{x}_{s}\right)_{\text {cal }}$ is defined as the pressure field for a source at $\mathbf{x}_{s}$ excited at $t=0$, and the receiver at $\mathbf{x}$ (model space) records the pressure field at $t$. Here, $\dot{p}$ represents the time derivative of $p, G\left(\mathbf{x},-t \mid \mathbf{x}_{r}, 0\right)$ is the Green's function associated with equation 1 for the velocity field $\mathbf{v}$, and $\delta \tau\left(\mathbf{x}_{r}, t \mid \mathbf{x}_{s}\right)$ is the back-propagated residual. The gradient $\mathbf{g}$ is formed by taking the zero-lag temporal correlation of the source-side wavefield $\dot{p}\left(\mathbf{x}, t \mid \mathbf{x}_{s}\right)$ with the back-propagated wavefield $\dot{p}\left(\mathbf{x}, t \mid \mathbf{x}_{r}\right)$. The source-side wavefield is calculated by exciting the source at $\mathbf{x}_{s}$ for each time step. As for the back-propagated wavefield, the observed seismogram $p\left(\mathbf{x}_{r}, t \mid \mathbf{x}_{s}\right)_{\text {obs }}$ at receiver $\mathbf{x}_{r}$ is weighted by its associated traveltime residual $\Delta \tau_{r s}$ (the difference between the observed and predicted traveltimes), and then back-propagated in reverse time, and recorded at $\mathbf{x}$ for each time step.

The WT workflow is shown in Figure 1a; it is used to iteratively find the velocity model that minimizes the objective function in equation 2 until the misfit change falls below a predefined tolerance. Here, we set the tolerance as 0.01 according to our experience.

\section{Ray-tracing traveltime tomography}

$\mathrm{RT}$ is based on the idea that the traveltime of a ray is the discretized integral of the slowness along the ray:

$$
t_{i}=\sum_{j=1}^{N} l_{i j} s_{j},
$$

where $t_{i}$ is the traveltime of the $i$ th ray, $l_{i j}$ is the segment length of the $i$ th ray that intersects the $j$ th cell, and $s_{j}$ is the slowness (reciprocal velocity) in the $j$ th cell. This results in an $M \times N$ system of equations, denoted in 
matrix-vector notation as $\mathbf{L s}=\mathbf{t}$, where $\mathbf{t}$ represents the recorded $M \times 1$ traveltime data vector, and $\mathbf{s}$ is the $N \times 1$ vector of unknown slownesses in the $N$ cells. The $M \times N$ matrix $L$ contains the segment lengths of the rays.

The least-squares solution of equation 9 is estimated by minimizing the following objective function:

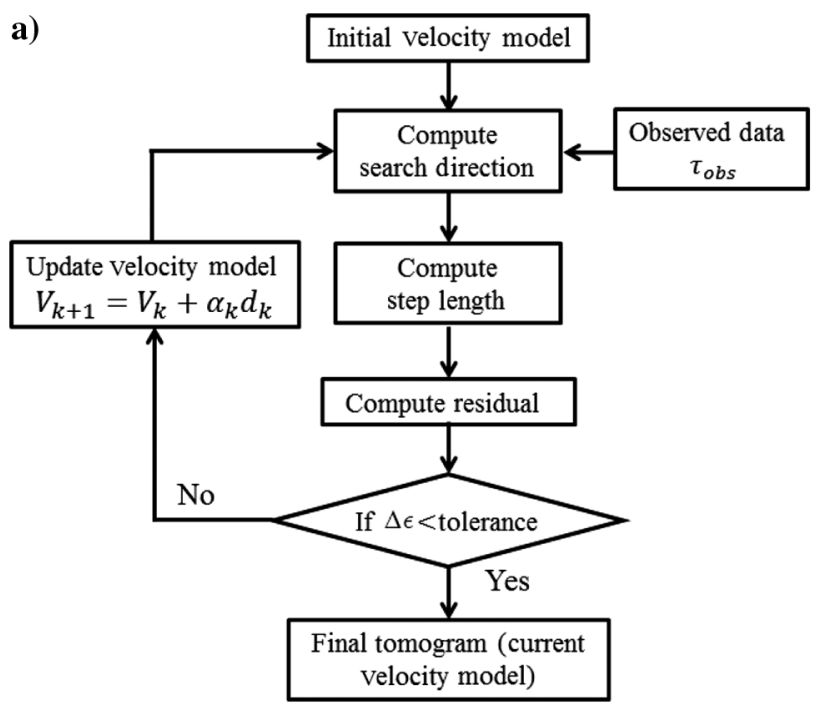

$$
\begin{aligned}
\epsilon & =\frac{1}{2}[\mathbf{L s}-\mathbf{t}]^{\top}[\mathbf{L s}-\mathbf{t}], \\
& =\frac{1}{2} \sum_{i=1}^{M} \sum_{j=1}^{N}\left(l_{i j} s_{j}-t_{i}\right)^{2}, \\
& =\frac{1}{2} \sum_{i=1}^{M} r_{i}^{2},
\end{aligned}
$$

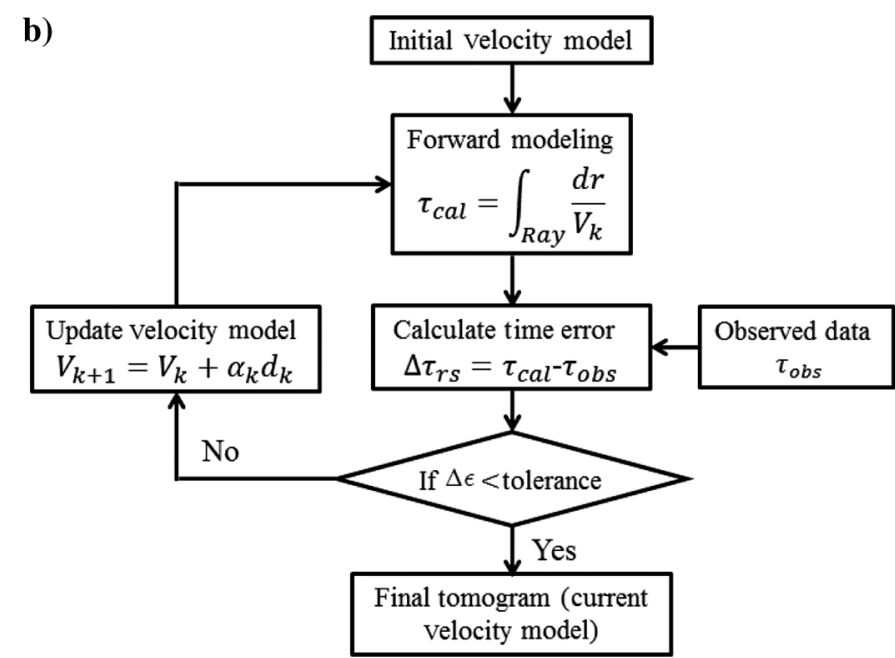

Figure 1. Flowchart for (a) WT inversion, where $\Delta \epsilon$ for WT is the misfit change percentage, and we set the tolerance as 0.01 . (b) RT inversion, where $\Delta \epsilon$ for RT is the rms residual, and the tolerance we set is a quarter of the dominant period.
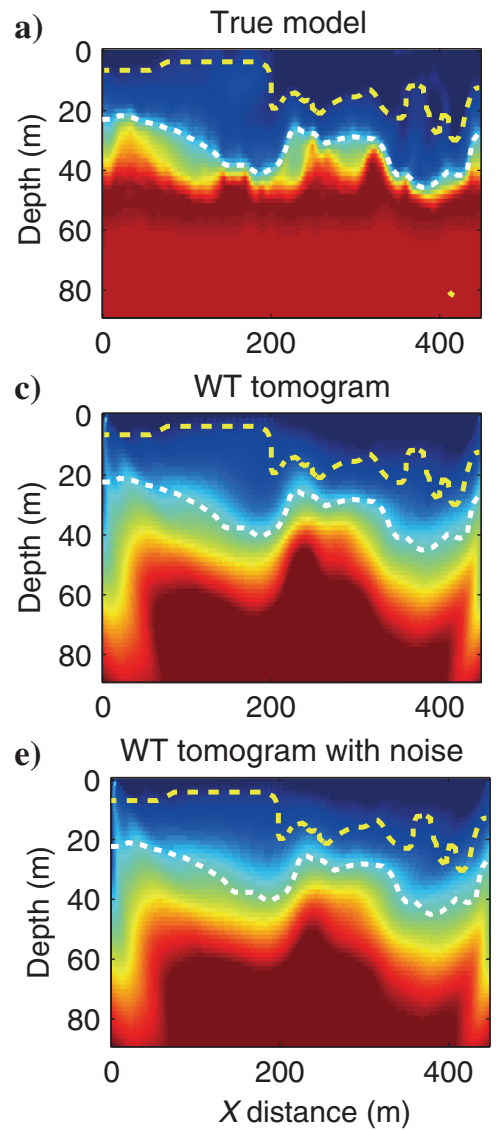
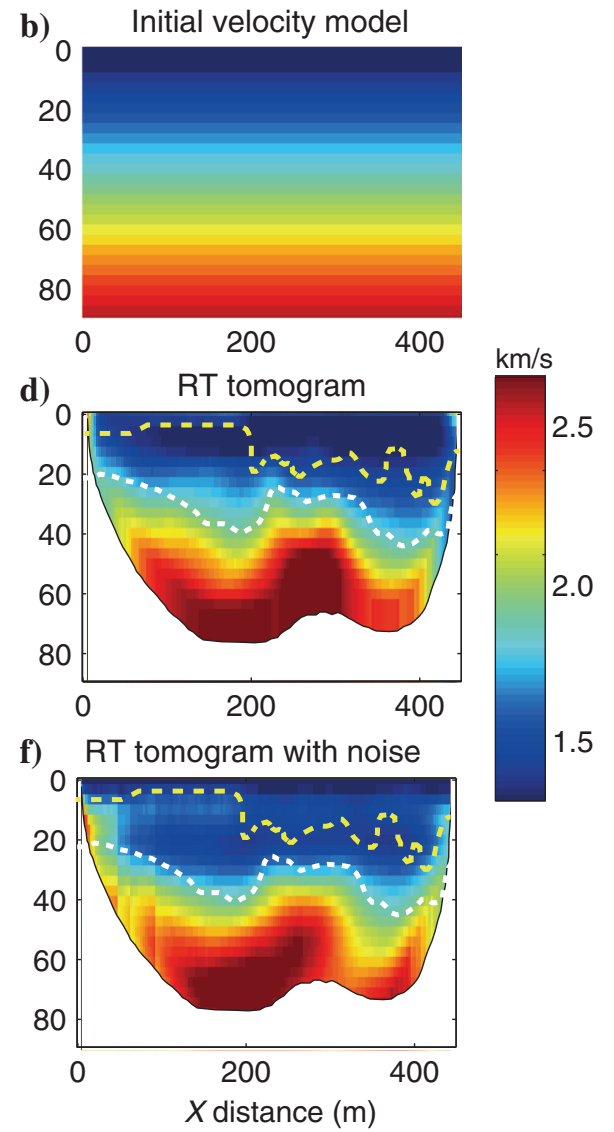

Figure 2. (a) The true velocity and (b) initial velocity models for the WT and RT methods, (c) WT, (d) RT, (e) WT tomograms with $10 \%$ random noise, and (f) RT tomogram with $10 \%$ random noise. For this velocity model, the characteristic horizontal variation of the velocity is $\approx 20 \mathrm{~m}$ along the interface for $25<z<45 \mathrm{~m}$. Thus, the high-frequency assumption of ray tracing is violated for a $50 \mathrm{~Hz}$ source wavelet having a wavelength approximately $40 \mathrm{~m}$ along this interface. The dashed yellow and white lines represent the velocity of 1550 and $1800 \mathrm{~m} / \mathrm{s}$, respectively, in the true model. 


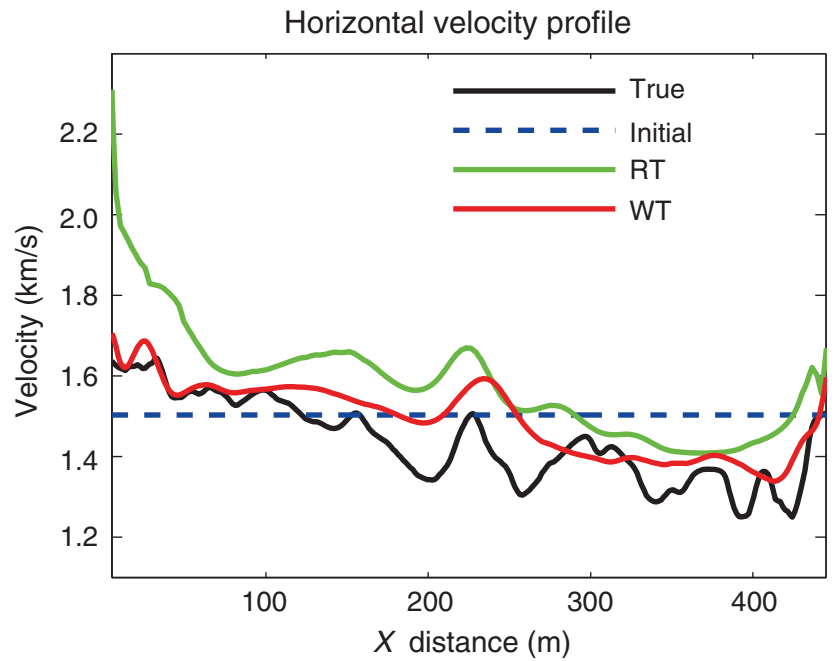

Figure 3. The comparison of the WT and RT velocity profiles at the depth of $25 \mathrm{~m}$, in which the black, blue, green, and red curves represent the true, initial, RT, and WT velocity profiles, respectively. This profile suggests that the WT method is more accurate than the RT method.

Table 1. The computational metrics for the WT and RT methods.

\begin{tabular}{lccc}
\hline & CPU time (min) & $\Delta \tau_{\text {rms }}(\mathrm{ms})$ & Iterations \\
\hline WT & 360 & 5.1 & 10 \\
RT & 3 & 5.8 & 30 \\
\hline
\end{tabular}

where $r_{i}=\sum_{j}\left(l_{i j} s_{j}-t_{i}\right)$ is the $i$ th traveltime residual between the predicted and observed $i$ th traveltimes. If the rays bend, then the matrix elements $\left(l_{i j}\right)$ depend on the unknowns $s_{j}$. In this case, the traveltime equations are nonlinear, so the rays must be recomputed for the new slowness model after each iteration. The slowness $\mathbf{s}$ can be iteratively updated using the conjugate-gradient method:

$$
\mathbf{s}_{k+1}=\mathbf{s}_{k}+\alpha_{k} \mathbf{d}_{k},
$$

where $\mathbf{s}_{k+1}$ is the slowness at the $(k+1)$ th iteration, $\alpha_{k}$ is the scale step length, and $\mathbf{d}_{k}$ is the updated direction determined by the derivative $\left(\partial \epsilon / \partial \mathbf{s}_{k}\right)$ of the objective function with respect to the model parameter. The workflow for implementing RT (Zhang and Toksöz, 1998) is described by the following steps (Figure 1b):

1) The first-arrival traveltimes $\left(\tau_{\text {obs }}\right)$ of the recorded data are picked.

2) An initial velocity model $\left(\mathbf{v}_{k}=\left(1 / \mathbf{s}_{k}\right), k=0\right)$ is given.

3) The first-arrival traveltimes $\left(\tau_{\text {cal }}\right)$ are calculated using the given initial velocity model.

4) If the root-mean-square (rms) error between the observed and the calculated traveltimes $\left(\tau_{\mathrm{obs}}-\tau_{\text {cal }}\right)$ is larger than a predefined tolerance (a quarter of the dominant period), then the velocity model is updated.

5) Steps 3 and 4 are iteratively repeated until the rms traveltime error is less than the given tolerance.
Figure 4. (a) Google map showing the location of the Wadi Qudaid experiment, (b) seismic survey line indicated by the blue double arrow line, (c) photograph showing the first layer composed of gravel and sand, with a thickness less than $5 \mathrm{~m}$, (d) a water well near the study area showing the second layer composed of sand and silt with some gravel, with the thickness of approximately 10-15 m, and the water table is at $z=18 \mathrm{~m}$, and (e) the seismic source is a $90 \mathrm{~kg}$ accelerated-weight drop.
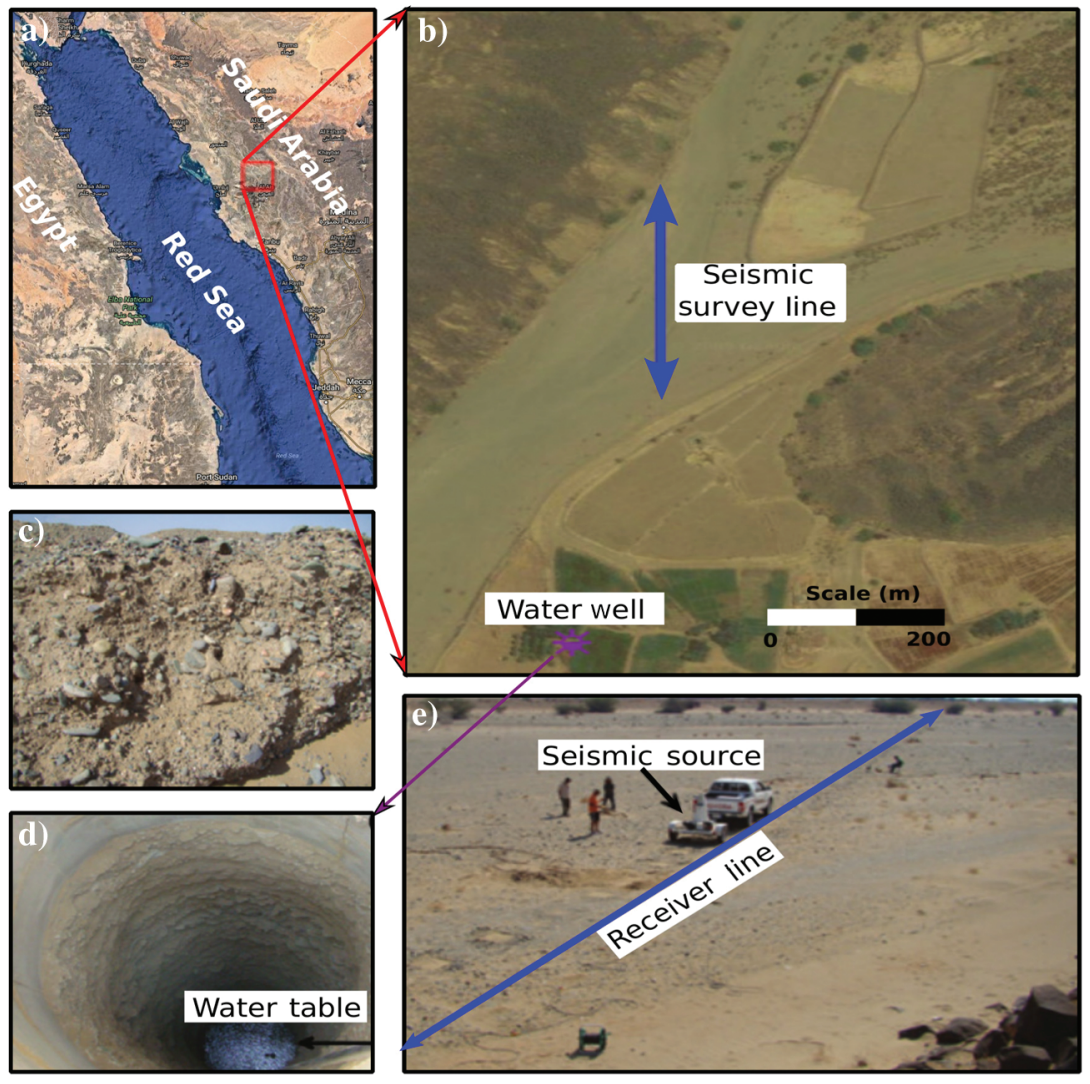


\section{Numerical results}

The WT and RT methods are now tested on one synthetic and two data sets recorded over the western side of Saudi Arabia. One of the seismic surveys was carried out at Wadi Qudaid for the purpose of determining the water-table depth. The other survey was carried out near the Gulf of Aqaba to detect the location of a hidden fault.

\section{Synthetic example}

The synthetic P-velocity model is shown in Figure 2a, which is a complex model with an irregular interface. It is discretized into $60 \times 300$ grid points with a grid size of $1.5 \mathrm{~m}$. A finite-difference solution to the $2 \mathrm{D}$ acoustic-wave equation is used to compute 60 shot gathers with 60 geophones evenly deployed on the free surface with a $7.5 \mathrm{~m}$ spacing. The source wavelet is a Ricker wavelet with a peak frequency of $50 \mathrm{~Hz}$. The goal of this synthetic test is to show that the WT method is more accurate than the RT method when its high-frequency assumption is violated. This violation occurs when the characteristic wavelength (the characteristic wavelength $\lambda^{\text {char }}=2 \pi / k^{\text {min }}$ of the earth's velocity variations is obtained by taking a 2D Fourier transform of the velocity model and identifying the minimum wavenumber $k^{\mathrm{min}}$ with significant amplitude) of the velocity model is about the same or is less than the dominant wavelength of the refraction wavefield (Luo and Schuster, 1991b). In this example the dominant wavelength of the refraction arrival is about $15 \mathrm{~m}$ in the first layer.

The initial velocity model is the gradient velocity model shown in Figure 2b. The traveltimes of the first arrivals are picked and inverted, and the resulting WT and RT tomograms are shown in Figure 2c and 2d,
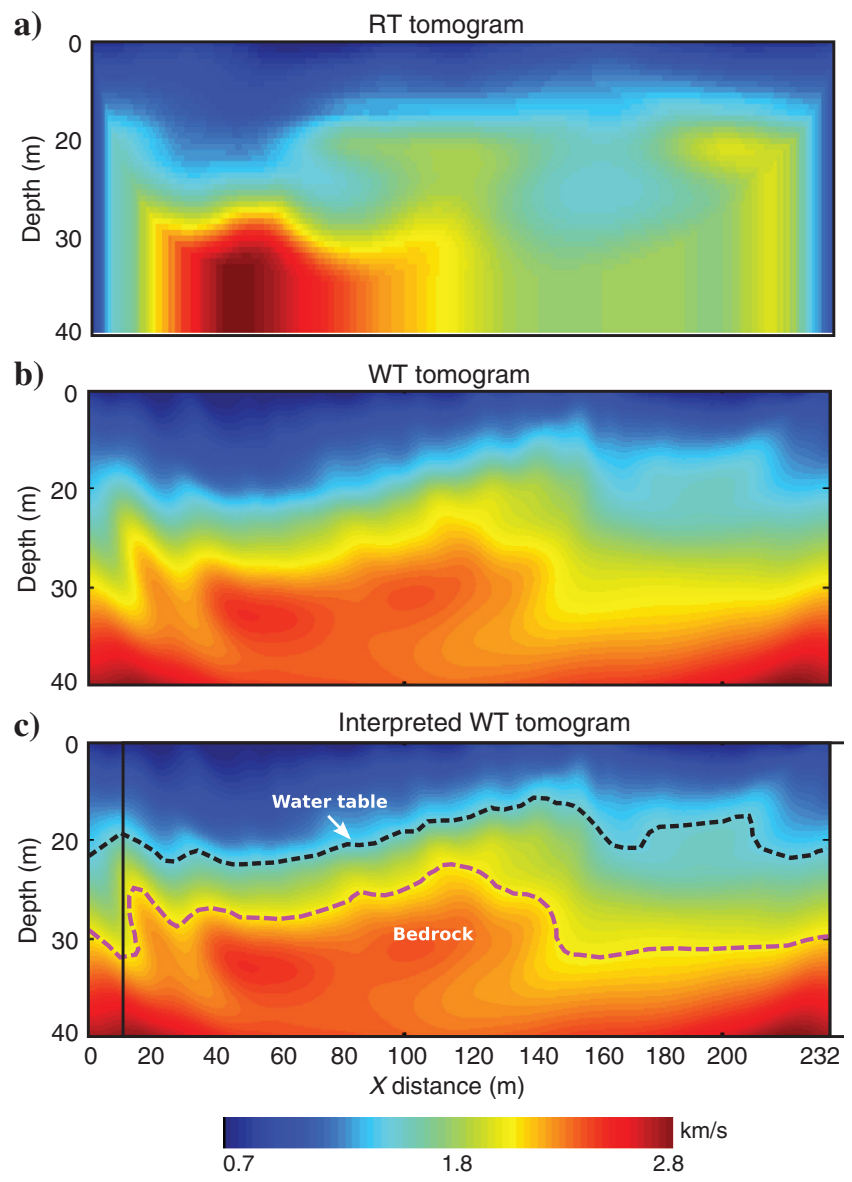

Figure 6. The RT and WT tomograms inverted from the Wadi Qudaid data: (a) RT, (b) WT, and (c) interpreted WT tomograms. The dashed black curve is the interpreted water table and the dashed pink curve is the interpreted bedrock interface.
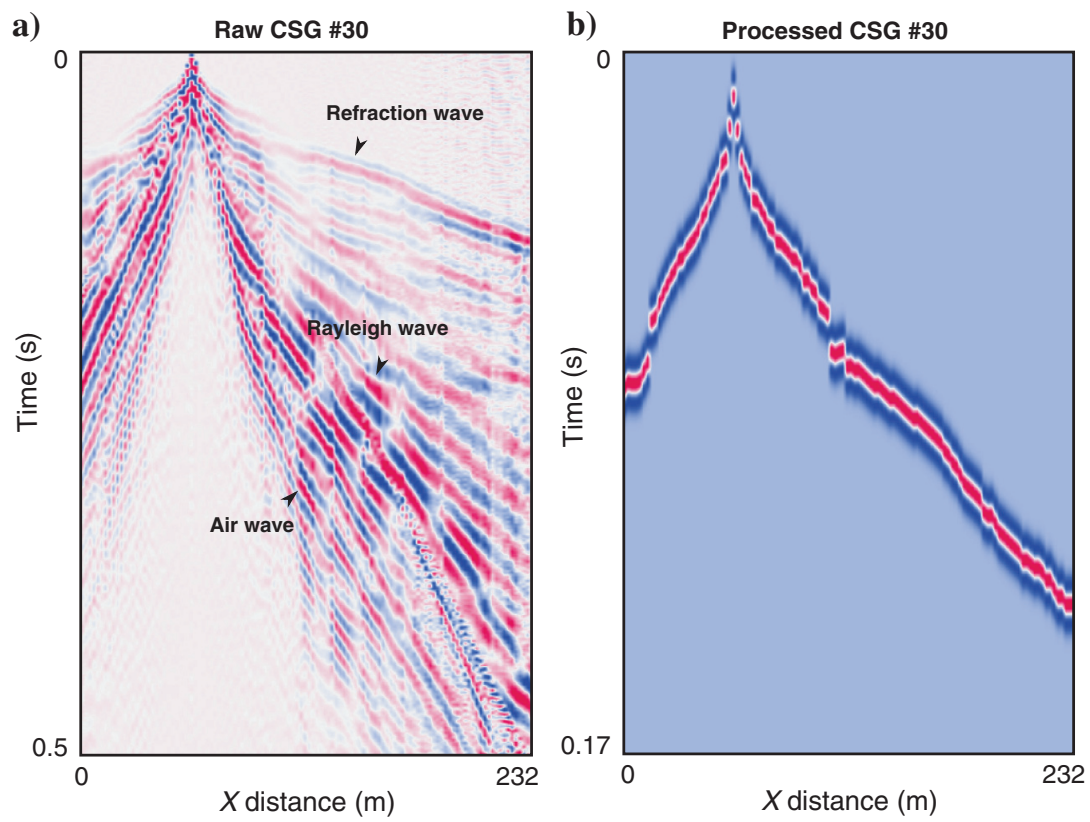

Figure 5. (a) CSG \#30, in which the air, Rayleigh, and refraction waves are indicated and (b) the processed CSG, in which only the refraction waves are extracted and processed. 
respectively. The dashed yellow and white lines denote the value of 1550 and $1800 \mathrm{~m} / \mathrm{s}$, respectively, in the actual tomogram, and they are used as the reference markers for the tomograms. In this regard, the WT tomogram is closer to the true model than the RT tomogram.

The horizontal velocity profiles at the depth of $25 \mathrm{~m}$ are shown in Figure 3, in which the black, blue, green, and red curves represent the velocity of the true model, initial model, RT tomogram, and WT tomogram, respectively. We can see that the velocity profile from the WT tomogram is more accurate than the RT profile. This suggests that the WT method can provide a more accurate velocity model than the RT method when the highfrequency assumption is violated.

Table 1 shows the computational metrics for the WT and RT methods. The rms traveltime differences for WT and RT are 5.1 and $5.8 \mathrm{~ms}$ at the final iteration, respectively. The WT method is 120 times more computationally expensive than the RT method using a 12-core workstation.

To test the noise sensitivity of these methods, $10 \%$ random noise is added to the raw wiggle traces for WT, and $10 \%$ random noise is added to the picked traveltimes for RT. The corresponding WT and RT tomograms with noisy data are shown in Figure 2e and 2f, respectively. We can see that the WT tomogram with noise (Figure 2e) is almost the same as the WT tomogram without noise (Figure 2c), whereas the RT tomogram with noise (Figure 2f) is different from the RT tomogram without noise (Figure 2d). This suggests that RT is more sensitive to the level of random noise in the input data.

\section{Data processing for field data}

The first-arrival traveltimes are manually picked, and a reciprocity test (reciprocity means that the traveltime from the shot location to the receiver location should be the same as the traveltime from the receiver location to the shot location) is applied to reject the traveltimes with picking errors larger than one quarter of the dominant period. Then, the traveltimes are used for the input data for RT. For WT inversion, to suppress elastic effects, such as surface waves, and account for geometric spreading, the seismic land data are processed by the following steps:

1) We transformed the $3 \mathrm{D}$ data to $2 \mathrm{D}$ data by multiplying the trace spectrum by $\sqrt{i / \omega}$ in the frequency domain (Boonyasiriwat et al., 2010).
Figure 7. (a) The traveltimes for shots 15, 65, 110 , in which the black, red and blue curves represent the observed, WT, and RT traveltimes, respectively, and (b) the normalized misfit functions for the WT and RT methods.

Figure 8. (a) Google map showing the location of the Aqaba survey, (b) photo showing an earthquake scarp (indicated by the dashed red line) on the surface, and the black arrow indicates the location of the seismic and resistivity survey lines.
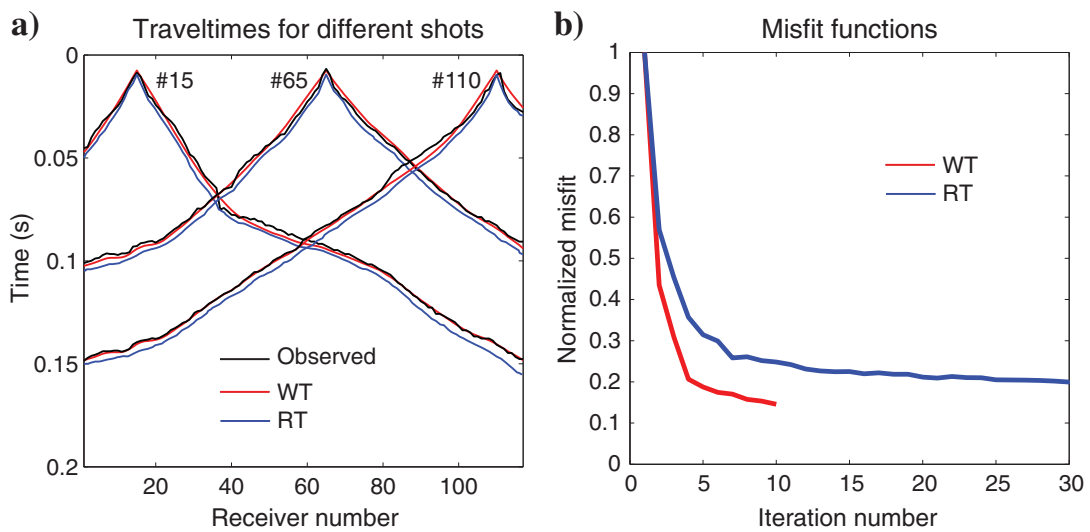

a)

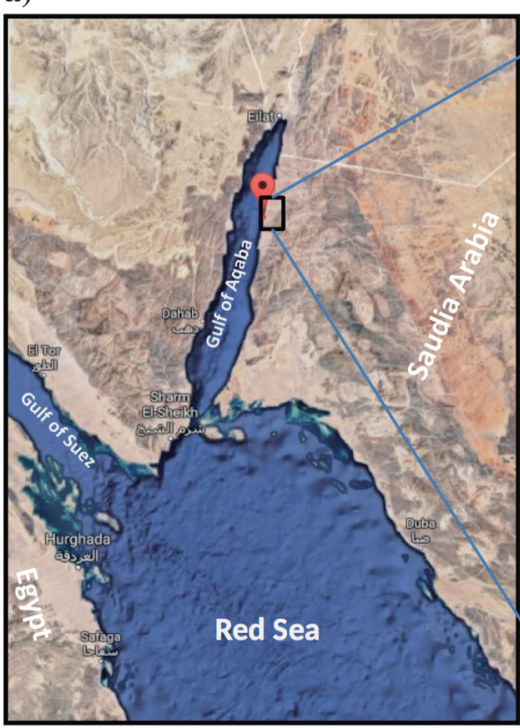

b)

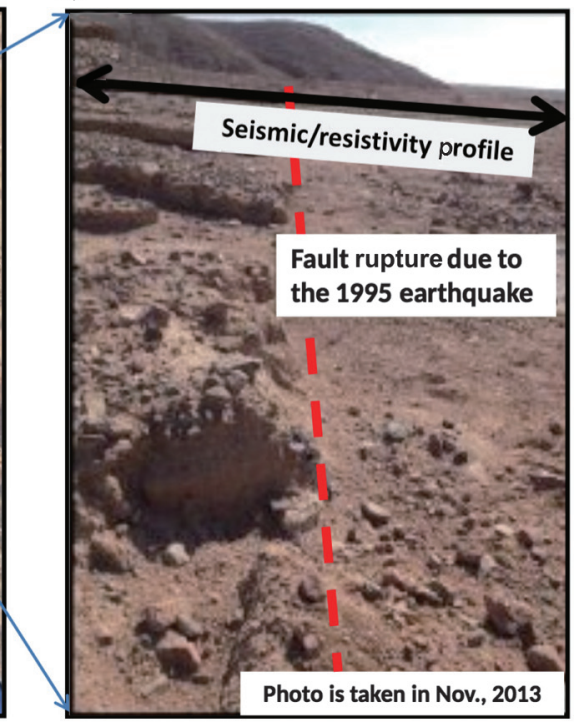


2) Partially correct for geometric spreading by multiplying the traces by $\sqrt{t}$ in the time domain.

3) The traces are windowed to only admit the firstarrival refraction events.

4) The traces recorded within one dominant wavelength from the source are muted because they contain surface waves and noise even after filtering.

\section{Wadi Qudaid field data}

A seismic survey was carried out at Wadi Qudaid (the red box in Figure 4a). The survey line is indicated by the double blue arrow, where 117 vertical-component geophones are deployed at $2.0 \mathrm{~m}$ intervals along the survey line. A $90 \mathrm{~kg}$ accelerated-weight drop (Figure 4e) is used as the source at every geophone position to record 117 common-shot gathers (CSGs). A water well near the study area shows that the near-surface sediments consist of two layers above the water table. The first layer is composed of gravel and sand with a thickness of less than $5 \mathrm{~m}$ (Figure 4c), the second layer is composed of sand and silt with some gravel with a thickness of approximately $10-15 \mathrm{~m}$, and the water table is approximately $18 \mathrm{~m}$ in depth (Figure 4d).

CSG \#30 is shown in Figure 5a, in which the air, Rayleigh, and refraction arrivals are marked. In our study, we only focus on the refraction arrivals, so all other events are muted (Figure 5b) and the result is used as the input to the WT inversion. The WT method can also be extended to invert the traveltimes of reflection arrivals (Zhang et al., 2011).

The initial model for WT is a velocity-gradient model, which is discretized into $160 \times 928$ grid points with the grid size of $0.25 \mathrm{~m}$. The RT, WT, and interpreted WT tomograms are shown in Figure $6 \mathrm{a}-6 \mathrm{c}$, respectively. For the near-surface zone from $0<z<25 \mathrm{~m}$, the RT and WT tomograms have a similar velocity structure, but the depth to the water table in the RT tomogram is deeper than in the WT tomogram. The dashed black curve (Figure $6 \mathrm{c}$ ) denotes the estimated water table according to the velocity contour value of $1550 \mathrm{~m} / \mathrm{s}$, which is consistent with the water well information in Figure $4 \mathrm{c}$ and $4 \mathrm{~d}$. The RT tomogram shows a high-velocity local anomaly at $20<x<110 \mathrm{~m}$ and $z>25 \mathrm{~m}$, which is not the case in the WT. In the WT tomogram, a continuous bedrock interface is shown at $25<z<35 \mathrm{~m}$.

Figure 7 a displays the traveltimes for shots 15,65 , and 110. The black, red, and blue curves represent the observed, WT, and RT traveltimes, respectively. We can see that the WT traveltimes are in better agreement with the observed traveltimes. Figure $7 \mathrm{~b}$ shows the normalized traveltime misfit functions for the WT and RT methods. The RT misfit function only decreases to $20 \%$ after 30 iterations; however, the WT misfit function decreases to approximately $13 \%$ after 10 iterations. In this example, the WT method has a better convergence rate than the $\mathrm{RT}$ method.

\section{Aqaba field data}

A seismic survey was carried out near the Gulf of Aqaba (the black box area in Figure 8a), in which 120

w CSG \#1

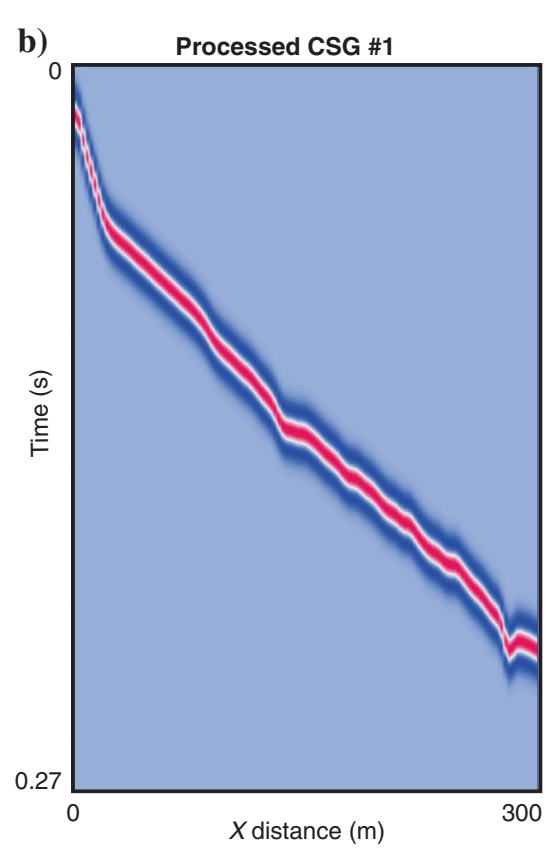

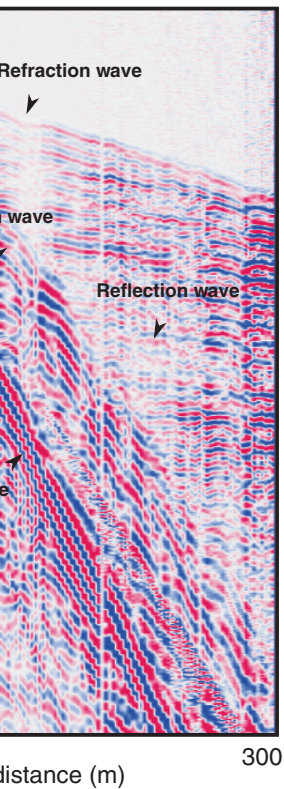

Figure 9. (a) CSG \#1, in which the air, Rayleigh, reflection, and refraction arrivals are indicated and (b) the processed CSG, in which only the refraction arrivals are extracted and processed.

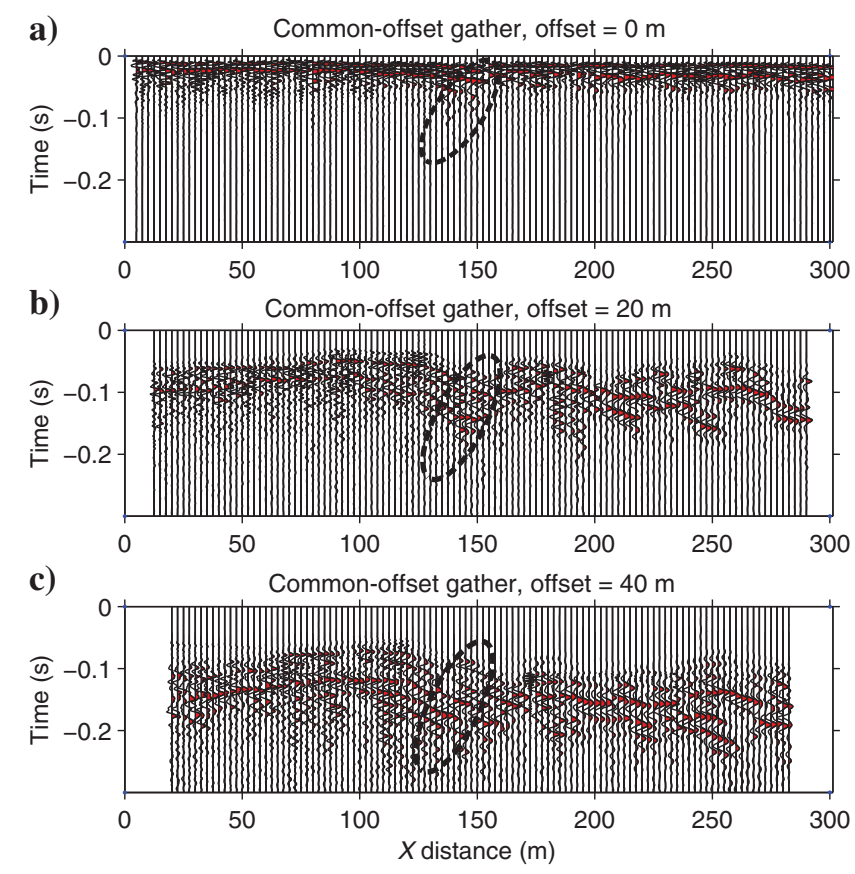

Figure 10. COGs for the source-receiver: (a) offset $=0 \mathrm{~m}$, (b) offset $=20 \mathrm{~m}$, and (c) offset $=40 \mathrm{~m}$. 
vertical-component geophones are deployed at $2.5 \mathrm{~m}$ intervals along the black double arrow line in Figure 8b. A $90 \mathrm{~kg}$ accelerated-weight drop is used for a source at every geophone position to record 120 CSGs. A 2D resistivity profile is acquired at the same location parallel to the seismic profile. The acquisition parameters of the resistivity profile are as follows:

1) number of electric nodes: 64

2) node interval: $5 \mathrm{~m}$

3) configuration array: Schlumberger-Wenner
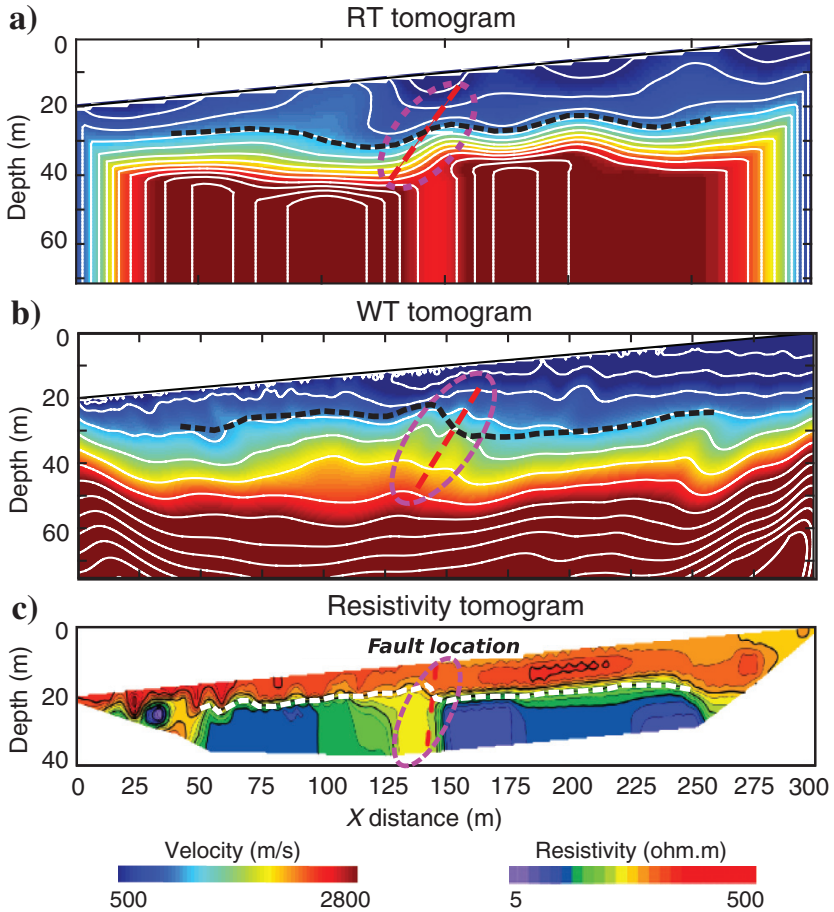

Figure 11. The tomograms inverted from the Aqaba data: (a) RT and (b) WT tomograms, in which the pink ellipses indicate possible fault locations and the dashed black curves represent the bedrock interfaces, (c) resistivity tomogram inverted by Res2DInv, a low-resistivity anomaly is shown at $130<x<145 \mathrm{~m}$, where the dashed white curve represents the bedrock interface. a) Traveltimes for different shots

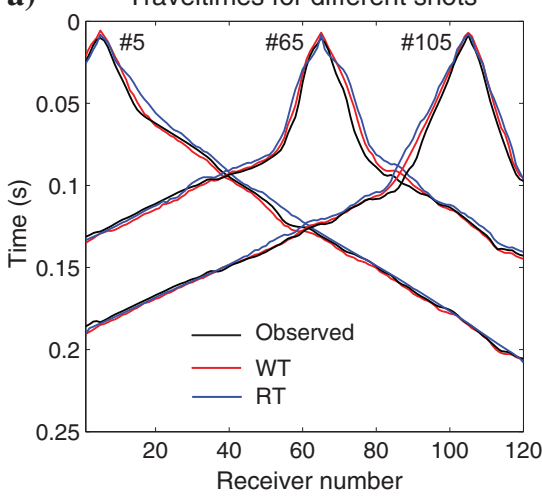

b) Misfit functions

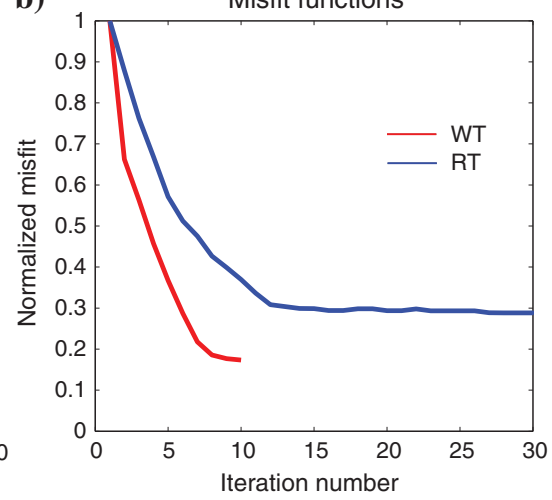

Figure 12. (a) The traveltimes for shots 5, 65, 105, in which the black, red, and blue curves are the observed, WT, and RT traveltimes, respectively. (b) The normalized misfit function for the WT and RT methods.
4) total profile length: $315 \mathrm{~m}$

5) the seismic and resistivity profiles share the same starting point of the profile.

A sample CSG is shown in Figure 9a. The air, Rayleigh, reflection, and refraction arrivals are indicated in Figure $9 \mathrm{a}$, and a processed CSG is shown in Figure 9b. The processed CSGs are the input data for the WT method.

We rearranged the raw CSGs into the common-offset gathers (COGs) with different offsets. Figure 10a shows the zero-offset COG, which shows a continuous horizontal event. Figure $10 \mathrm{~b}$ and $10 \mathrm{c}$ shows the COGs with the offsets of 20 and $40 \mathrm{~m}$, respectively, in which the events are no longer continuous everywhere, especially at $x=145 \mathrm{~m}$ (the area indicated by the dashed black ellipse). The time delays of the surface waves in the distorted area (ellipses in Figure 10b and 10c) imply that the shear velocity is slower, and suggest the location of a possible fault.

The initial P-velocity model for WT inversion is a gradient velocity model, which is discretized into $150 \times 598$ grid points with a grid size of $0.5 \mathrm{~m}$. The RT and WT tomograms after 10 iterations are shown in Figure 11a and 11b. The RT and WT tomograms are similar to each other. The RT and WT tomograms suggest the hidden fault location (indicated by the pink ellipse and the dashed red line) is at $125<x<145 \mathrm{~m}$, which is consistent with the resistivity tomogram shown in Figure 11c. However, compared with the RT tomogram (Figure 11a), the bedrock interface indicated by the dashed black curve in the WT tomogram (Figure 11b) is more consistent with the bedrock interface revealed by the resistivity tomogram (Figure 11c).

The recorded resistivity data were inverted using the Res2DInv software to generate the resistivity tomogram in Figure 11c. Two distinct layers are visible in the resistivity tomogram, the first layer has resistivity values ranging from 250 to $500 \mathrm{ohm}-\mathrm{m}$ with the layer thickness ranging between 6 and $10 \mathrm{~m}$. The second layer extends to the bottom of the section and has low-resistivity values ranging between 10 and 50 ohm-m, except between offsets 130 and $145 \mathrm{~m}$, in which the resistivity values appear to increase to approximately $250 \mathrm{ohm}-\mathrm{m}$. The location of the fault is shown on the resistivity tomogram as a vertical anomaly (offsets between 130 and $145 \mathrm{~m}$ ) with higher resistivity values (250 ohm.m) shown in Figure 11c.

Figure 12a shows the traveltimes for shots 15, 65, and 110. The black, red, and blue curves represent the observed, WT, and RT traveltimes, respectively. We can see that the WT traveltimes are in better agreement with the observed traveltimes. Figure $12 \mathrm{~b}$ shows the normalized misfit functions for the WT and RT methods. The misfit function for the RT method only decreases to $25 \%$ after 30 iterations; however, the WT misfit func- 
tion decreases to approximately $16 \%$ after 10 iterations. In this example, the WT method has a better convergence rate than the $\mathrm{RT}$ method.

\section{Conclusion}

The WT and RT methods are applied to one synthetic example and two seismic land data sets acquired in western Saudi Arabia. The goal of the Wadi Qadid experiment is to determine the topography of the water table, and the goal of the other survey is to locate the Aqaba fault. The seismic field data sets are carefully processed using geometric spreading corrections, shot normalization, window muting along the first arrivals, and muting near-offset traces. The WT method generates P-velocity tomograms, from which we can clearly identify the water-table depth in the Wadi Qudaid experiment, and the fault location in the Aqaba experiment.

Compared with the RT tomogram, the WT tomogram from Wadi Qudaid indicates a more accurate water table depth that is consistent with the water-well information. The WT tomogram from the Aqaba data suggests a more accurate hidden fault location, which is in closer agreement with the resistivity tomogram. Although the WT method requires more than an order-of-magnitude more computational time compared with the RT method, it can provide a more accurate initial velocity model for MEWI or FWI.

\section{Acknowledgments}

The research reported in this publication was supported by the King Abdullah University of Science and Technology (KAUST) in Thuwal, Saudi Arabia. We are grateful to the sponsors of the Center for Subsurface Imaging and Modeling Consortium for their financial support. For computer time, this research used the resources of the Supercomputing Laboratory at KAUST and the IT Research Computing Group. We thank them for providing the computational resources required for carrying out this work.

\section{References}

Aki, K., A. Christoffersson, and E. S. Husebye, 1977, Determination of the three dimensional seismic structure of the lithosphere: Journal of Geophysical Research, 82, 277-296, doi: 10.1029/JB082i002p00277.

Bai, J., D. Yingst, R. Bloor, and J. Leveille, 2014, Viscoacoustic waveform inversion of velocity structures in the time domain: Geophysics, 79, no. 3, R103-R119, doi: 10.1190/geo2013-0030.1.

Boonyasiriwat, C., G. T. Schuster, P. Valasek, and W. Cao, 2010, Applications of multiscale waveform inversion to marine data using a flooding technique and dynamic early-arrival windows: Geophysics, 75, no. 6, R129R136, doi: 10.1190/1.3507237.

Frazer, L. N., and X. Sun, 1998, New objective functions for waveform inversion: Geophysics, 63, 213-222, doi: 10 $.1190 / 1.1444315$.

Humphreys, E., R. W. Clayton, and B. H. Hager, 1984, A tomographic image of mantle structure beneath southern
California: Geophysical Research Letters, 11, 625-627, doi: 10.1029/GL011i007p00625.

Luo, Y., and G. T. Schuster, 1991a, Wave equation inversion of skeletalized geophysical data: Geophysical Journal International, 105, 289-294, doi: 10.1111/j.1365-246X .1991.tb06713.x.

Luo, Y., and G. T. Schuster, 1991b, Wave-equation traveltime inversion: Geophysics, 56, 645-653, doi: 10.1190/1.1443081.

Neelamani, R., A. I. Baumstein, D. G. Gillard, M. T. Hadidi, and W. L. Soroka, 2008, Coherent and random noise attenuation using the curvelet transform: The Leading Edge, 27, 240-248, doi: 10.1190/1.2840373.

Scales, L., 1985, Introduction to non-linear optimization: Springer-Verlag Inc.

Schuster, G. T., 2017, Seismic inversion: SEG.

Vermeer, G. J., 2002, 3D seismic survey design: SEG.

Wright, S., and J. Nocedal, 1999, Numerical optimization: Springer Science, 67-68.

Yilmaz, Ö., 2001, Seismic data analysis: Processing, inversion, and interpretation of seismic data: SEG.

$\mathrm{Yu}, \mathrm{H}$., and S. M. Hanafy, 2014, An application of multiscale early arrival waveform inversion to shallow seismic data: Near Surface Geophysics, 12, 549-557, doi: 10 .3997/1873-0604.2014002.

Zhang, J., and M. N. Toksöz, 1998, Nonlinear refraction traveltime tomography: Geophysics, 63, 1726-1737, doi: 10 .1190/1.1444468.

Zhang, S., G. Schuster, and Y. Luo, 2011, Wave-equation reflection traveltime inversion: 81st Annual International Meeting, SEG, Expanded Abstracts, 2705-2710.

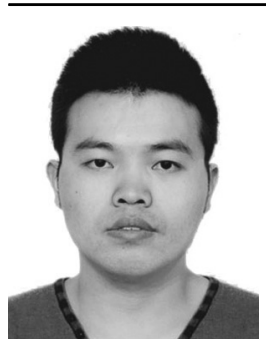

Lei Fu received a B.S. (2009) from China University of Geoscience (Wuhan) and a Ph.D. (2014) in geophysics from JiLin University, China. He joined JiLin University as a lecturer (2014-now), then joined King Abdullah University of Science and Technology (KAUST) as a postdoc in 2015. His research interests include seismic inversion and ground-penetrating radar.

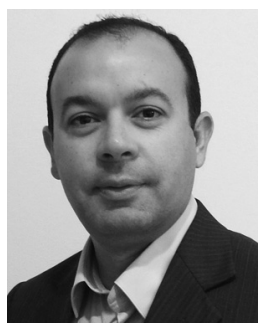

Sherif M. Hanafy received a B.S. (1993) and an M.S. (1996) from Cairo University, Egypt, and a Ph.D. (2002) in geophysics from Kiel University, Germany - Cairo University, Egypt. He joined Cairo university as a teacher assistant (1993-2002), assistant professor (2002-2010), and associate professor (2010-now). He had two postdocs at the University of Utah (2004-2005) and (2007-2009), then he joined King Abdullah University of Science and Technology (KAUST) as a senior research scientist in 2009. His research interests include environmental and engineering applications of geophysics, interferometry, and tomography. He is member of SEG, EEGS, EAGE, and IOP. 